\title{
Human islet distribution programme for basic research: activity over the last 5 years
}

\author{
Rita Nano • Domenico Bosco • Julie A. Kerr-Conte • Marie Karlsson • \\ Solange Charvier • Raffaella Melzi • Rimed Ezzouaoui • \\ Alessia Mercalli • Albert Hwa • François Pattou • Olle Korsgren • \\ Thierry Berney • Lorenzo Piemonti
}

Received: 7 January 2015 / Accepted: 27 January 2015 / Published online: 21 February 2015

(C) Springer-Verlag Berlin Heidelberg 2015

Keyword Human pancreatic islet

\section{Abbreviation \\ ECIT European Consortium for Islet Transplantation}

To the Editor: Single-centre or collaborative efforts have been made to provide human pancreatic islets for use in basic science research. These include the Integrated Islet Distribution Program in the USA [1], the Human Islet Distribution Program for Basic Research at the University of Alberta in Canada [2] and the Oxford Islet Transplant Program in the UK. The European Consortium for Islet Transplantation (ECIT) set up the human islet distribution programme for basic research (http://ecit.dri-sanraffaele.org/en/activeprogram/index.html) in 2006 to distribute high-quality human islets to the European diabetes research community. The programme currently involves the San Raffaele Scientific Institute (Milan, Italy), the Hôpitaux Universitaires

R. Nano $\cdot$ R. Melzi $\cdot$ A. Mercalli $\cdot$ L. Piemonti $(\bowtie)$

Diabetes Research Institute, IRCCS San Raffaele Scientific Institute, Via Olgettina 60, 20132 Milan, Italy

e-mail: piemonti.lorenzo@hsr.it

D. Bosco $\cdot$ S. Charvier $\cdot$ T. Berney

Hôpitaux Universitaires de Genève, Genève, Switzerland

J. A. Kerr-Conte $\cdot$ R. Ezzouaoui $\cdot$ F. Pattou

European Genomic Institute for Diabetes, Lille University Hospital, Lille, France

M. Karlsson · O. Korsgren

Uppsala University Hospital, Uppsala, Sweden

A. Hwa

JDRF, New York, NY, USA de Genève, (Genève, Switzerland), Uppsala University Hospital (Uppsala, Sweden) and the Centre Hospitalier Regional Universitaire de Lille (France). The programme is coordinated by the San Raffaele Diabetes Research Institute and receives financial support from the JDRF. A web-based platform was developed in 2009 to coordinate and track programme activity (http://ecit.dri-sanraffaele.org/en/register/ index.html). Islet isolation and shipment were performed according to centre protocols. Here we report on programme activity over the past 5 years (from 1 November 2009 to 31 October 2014).

One-hundred and twenty research groups have completed the online registration process. Of these, 66 users have made at least one application to receive islets. A total of 93 applications were submitted from 13 countries: Switzerland $(n=26)$, Sweden $(n=13)$, Israel $(n=13)$, Germany $(n=12)$, France $(n=9)$, Italy $(n=7)$, Spain $(n=4)$, Denmark $(n=3)$, UK $(n=2)$ and one each from Austria, Norway, Ireland and Finland. The total number of islets requested was 106.187 million (Fig. 1a). An ideal islet purity of $\geq 70 \%$ was requested in $97 \%$ of the applications. In terms of applications from individual research groups, the median number of requested islets was $20 \times 10^{3}$ (IQR $12.5 \times 10^{3}$ ) in 20 (IQR 37.5) shipments. Over the years the requests for human pancreatic islets from the programme for use in basic science research have increased substantially, with the number of islets requested reaching 31.042 million in 2014. The designated use of the islets in basic science, as stated on the request form to the ECIT centres, are shown in Fig. 1c, categorised into different areas of research.

Over the first 5 years of the programme, a total of 31.643 million human islets have been distributed throughout 1,130 shipments (Fig 1a,b). The production and delivery of islets by the ECIT centres have been stable over the years. However, because of the burgeoning demand for islets and the lack of funding to cover additional islet isolation procedures, the 
a
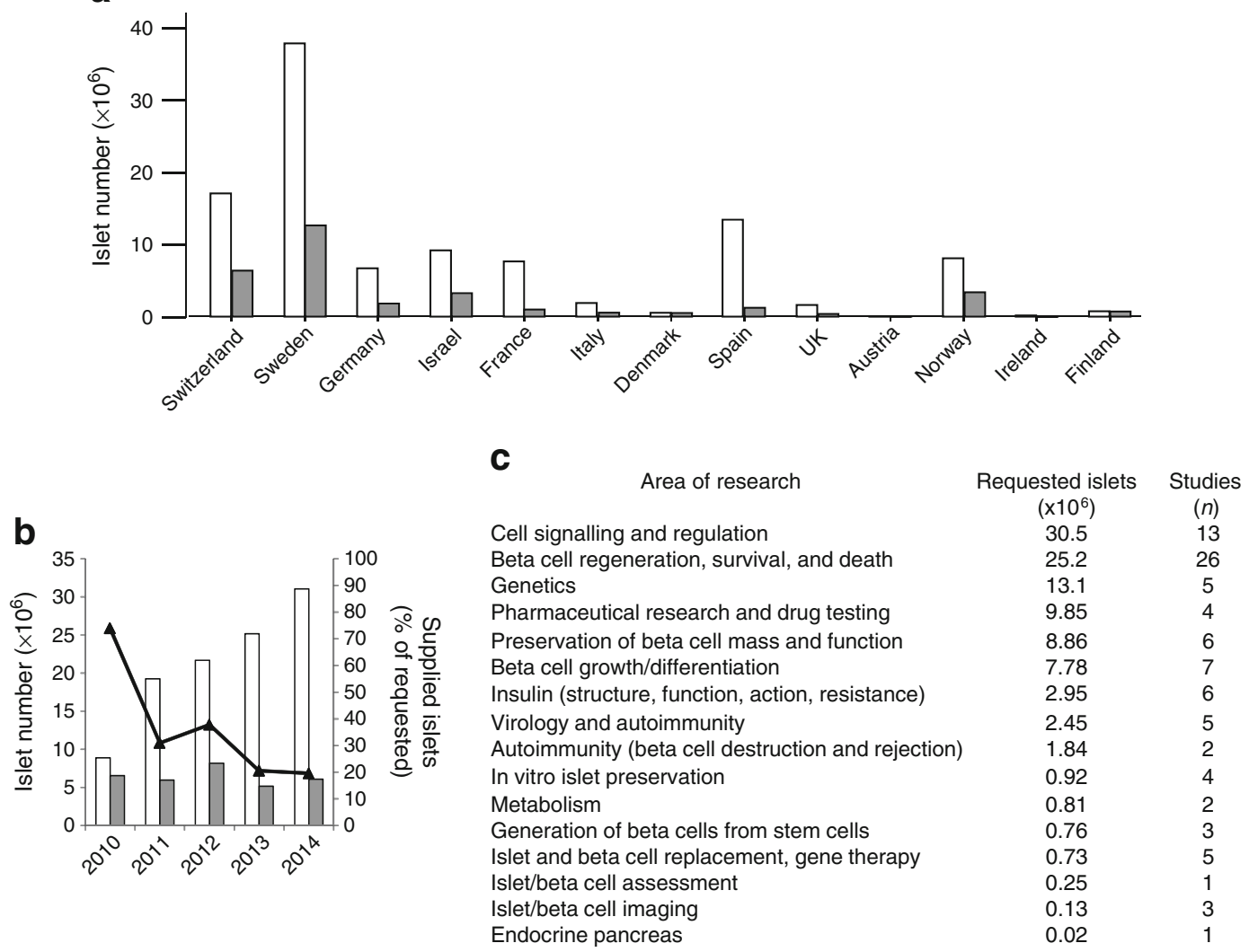

Fig. 1 Requests for islets from the Human Islet Distribution Program for Basic Research. From November 2009 though to the end of October 2014, a total of 93 applications were submitted. (a) Requested (white bar) and supplied (grey bar) islets according to country. (b) Requested (white bar) and supplied (grey bar) islets according to year of application. The black line represents the percentage of requests supplied. (c) Table of applications to the ECIT consortium according to major area of research

Research activity related to beta cell biology has drastically increased over recent years, leading to a significant rise in the demand for human pancreatic islets $[1,3]$. This is because human pancreatic islets are still the gold standard for the assessment of beta cell function, even though a variety of surrogate alternatives have been suggested, including non-human pancreatic islets or rodent/human beta cell lines [4-7]. Even though molecular, cellular and genetic data have been collected from these surrogate models and have proved useful for certain aspects of beta cell maturation and function, many studies have shown species-specific differences in islets (especially between human and rodent models) and line-specific differences in islet-like insulinproducing cells obtained from germ-cell precursors when examining proliferative potential, differentiation capacity, response to injury, composition and organisation of cell clusters, and type and amount of secreted products. Based on the experience of the human islet distribution programme for basic research in Europe and of similar islet distribution programmes, it has become evident that sufficient supply and further 
stimulation of translational research is critically dependent on an enhanced availability of high-quality and high-purity human islet preparations $[3,8]$. The 5 year analysis of our activity highlights one critical issue: the expanding gap between the demand for islets and the supply available. Although the programme has so far succeeded in meeting at least a small proportion of the islet demand, it is very likely that in the next few years this will not be the case. The situation may be about to worsen for two reasons. The sustained improvement in the isolation procedure, combined with the progressive increase of isolation costs, will reduce the proportion of preparations unsuitable for the clinic, further restricting the availability of islets for research. Moreover, the European Commission will soon award the first grants of the Horizon 2020 programme 'Biomaterial for the treatment of diabetes mellitus' (http://ec.europa.eu/ research/participants/portal/desktop/en/opportunities/ h2020/topics/2511-nmp-10-2014.html), and this funding will generate a dramatic increase in human islet demand. The ECIT human islet distribution programme for basic research was established to help address the supply and demand issues faced by islet isolation laboratories and basic scientists. Over the first 5 years of the programme, islets have been provided free of charge. Although this distribution network has had a global influence on diabetes research, in the future it will likely face economic hurdles to providing human islets to a growing research community.

Acknowledgements This research was supported by the Juvenile Diabetes Research Foundation International [1-RSC-2014-100-I-X (Geneva); 1-RSC-2014-101-I-X (Lille); 1-RSC-2014-90-I-X (Milan), 1-RSC-2014-99-I-X (Uppsala)]. We thank Marina Scavini (Diabetes Research Institute, IRCCS San Raffaele Scientific Institute, Milan) for manuscript editing.
Funding This research was supported by JDRF International [1-RSC2014-100-I-X (Geneva); 1-RSC-2014-101-I-X (Lille); 1-RSC-2014-90I-X (Milan), 1-RSC-2014-99-I-X (Uppsala)].

Duality of interest The authors declare that there is no duality of interest associated with this manuscript.

Contribution statement RN, DB, JAK-C, MK, SC, RM, RE, AM, FP, $\mathrm{OK}$ and $\mathrm{TB}$ performed islet isolation, provided data and contributed to the discussion. RN, AH and LP developed the concept, analysed the data, wrote the manuscript, promoted the study and researched data. All authors approved the version submitted for publication. LP is the guarantor of this work.

\section{References}

1. Kaddis JS, Olack BJ, Sowinski J, Cravens J, Contreras JL, Niland JC (2009) Human pancreatic islets and diabetes research. JAMA 301: $1580-1587$

2. Kin T, O'Gorman D, Schroeder A et al (2011) Human islet distribution program for basic research at a single center. Transplant Proc 43:31953197

3. Chakradhar S (2014) Diabetes researchers fear worsening access to human islets. Nat Med 20:567

4. Narushima M, Kobayashi N, Okitsu T et al (2005) A human beta-cell line for transplantation therapy to control type 1 diabetes. Nat Biotechnol 23:1274-1282

5. Ravassard P, Hazhouz Y, Pechberty S et al (2011) A genetically engineered human pancreatic beta cell line exhibiting glucoseinducible insulin secretion. J Clin Invest 121:3589-3597

6. Pagliuca FW, Millman JR, Gurtler M et al (2014) Generation of functional human pancreatic beta cells in vitro. Cell 159:428439

7. Rezania A, Bruin JE, Arora P et al (2014) Reversal of diabetes with insulin-producing cells derived in vitro from human pluripotent stem cells. Nat Biotechnol 32:1121-1133

8. Kulkarni RN, Stewart AF (2014) Summary of the keystone islet workshop (April 2014): the increasing demand for human islet availability in diabetes research. Diabetes 63:3979-3981 